Polymer Journal, Vol. 38, No. 10, pp. 1043-1054 (2006)

(C) 2006 The Society of Polymer Science, Japan

\title{
Soluble Polyimides Based on Diaminobenzoic Acid Alkylester
}

\author{
Yusuke TsudA, ${ }^{1, \dagger}$ Manami KoJIMA, ${ }^{2}$ and Jae Min $\mathrm{OH}^{3}$ \\ ${ }^{1}$ Department of Biochemistry \& Applied Chemistry, Kurume National College of Technology, \\ 1-1-1 Komorino, Kurume 830-8555, Japan \\ ${ }^{2}$ Advanced Engineering School, Kurume National College of Technology, \\ 1-1-1 Komorino, Kurume 830-8555, Japan \\ ${ }^{3}$ Electronic Chemical Material Division, SAMSUNG Cheil Industries Inc., \\ 332-2, Gochun-Dong, Euiwang-Shi, Kyoungki-Do 437-010, Korea
}

(Received March 28, 2006; Accepted July 20, 2006; Published August 30, 2006)

\begin{abstract}
The synthesis and characterization of a novel series of soluble polyimides, that are based on diaminobenzoic acid alkylester (DBAE) having long-chain alkyl groups with 8-14 carbon atoms, are described. Polyimides obtained from 3,3',4,4'-benzophenonetetracarboxylic dianhydride (BTDA) and DBAE-8-14, and copolyimides based on BTDA, DBAE-8-14, and 4,4'-diaminodiphenylether (DDE) were insoluble in polar solvents such as $N$-methyl-2pyrrolidone (NMP) and the effect of long-chain linear alkyl groups for the enhancement of solubility was not recognized. However, it was found that two methods improve the solubility. One is the use of branched alkyl groups such as nonan-5-yl and 2,6-dimethylheptane-4-yl, and the other is the use of relatively flexible diamine co-monomer, 4,4'diaminodiphenylmethane (DDM). These polyimides and copolyimides based on DBAE were soluble in various polar solvents and exhibited good thermal stability in air and under nitrogen. The effect of DBAE for the enhancement of solubility was lower than the effects of alkyloxydiaminobenzene (AODB) and alkyldiaminobenzophenone (ADBP), probably due to the rigid ester linkage group in DBAE. [doi:10.1295/polymj.PJ2005257]

KEY WORDS Polyimide / Soluble Polyimide / Diaminobenzamide Alkylester / Branched Alkyl Group / Copolymerization / Thermal Stability / Solubility /
\end{abstract}

Polyimides exhibit excellent thermal and mechanical properties, and have extensive engineering and microelectronics applications. ${ }^{1}$ Aromatic polyimides such as pyromellitic polyimides are prepared from aromatic diamines and aromatic tetracarboxylic dianhydrides via poly(amic acids). Since conventional aromatic polyimides are insoluble, these polymers are usually processed as the corresponding soluble poly(amic acid) precursors, and then either thermally or chemically imidized. However, owing to the instability of poly(amic acids) and the liberation of water in the imidization process, problems can arise. Extensive research has been recently carried out to improve the solubility of polyimides. ${ }^{2-24}$ We have reported the synthesis and characterization of soluble polyimides and copolyimides based on alicyclic dianhydride such as 2,3,5-tricarboxycyclopentyl acetic dianhydride (TCA-AH), 5-(2,5-dioxotetrahydrofuryl)-3-methyl-3cyclohexene-1,2-dicarboxylic anhydride (cyclohexene-DA), and 4-(2,5-dioxotetrahydrofuran-3-yl)-tetralin-1,2-dicarboxylic anhydride (tetralin-DA). ${ }^{25-27}$ Conventional tetracarboxylic dianhydrides such as 3,3',4,4'-benzophenone tetracarboxylic dianhydride (BTDA) and alkyldiaminobenzophenone (ADBP) having long-chain linear alkyl groups with 9-14 carbon atoms (ADBP-9-14), ${ }^{28}$ and BTDA and alkyloxy- diaminobenzene (AODB) having long-chain linear alkyl groups with 10-14 carbon atoms (AODB-1014) have also been used (Scheme 1). ${ }^{29}$ It was concluded that ADBP with an even number of carbon atoms was effective in enhancing the solubility, while polymers based on ADBP with an odd number of carbon atoms remained insoluble. AODB bearing alkyl groups via an ether linkage are more effective in comparison with ADBP where these groups are attached by benzoyl groups, thus, all polyimides based on BTDA and AODB-10-14 were soluble. Copolymerization using the conventional aromatic diamine, DDE resulted in the improvement of both the molecular weight and the thermal stability, and demonstrated that AODB-12 was the most effective in enhancing the solubility of the AODB-10-14-based polyimides. It can be presumed that the entropy effect of longchain linear alkyl groups increases up to 12 methylene units, however, the crystallization of these side groups having more than 12 methylene units might decrease the solubility of the polymers. The recent our papers have described the soluble polyimides having dendric moieties on their side chain, ${ }^{30}$ and the synthesis of soluble polyimides in ionic liquids was also investigated. ${ }^{31}$

This paper reports the synthesis and characteriza-

${ }^{\dagger}$ To whom correspondence should be addressed (Tel: +81-942-35-9408, Fax: +81-942-35-9329, E-mail: tsuda@kurume-nct.ac.jp). 

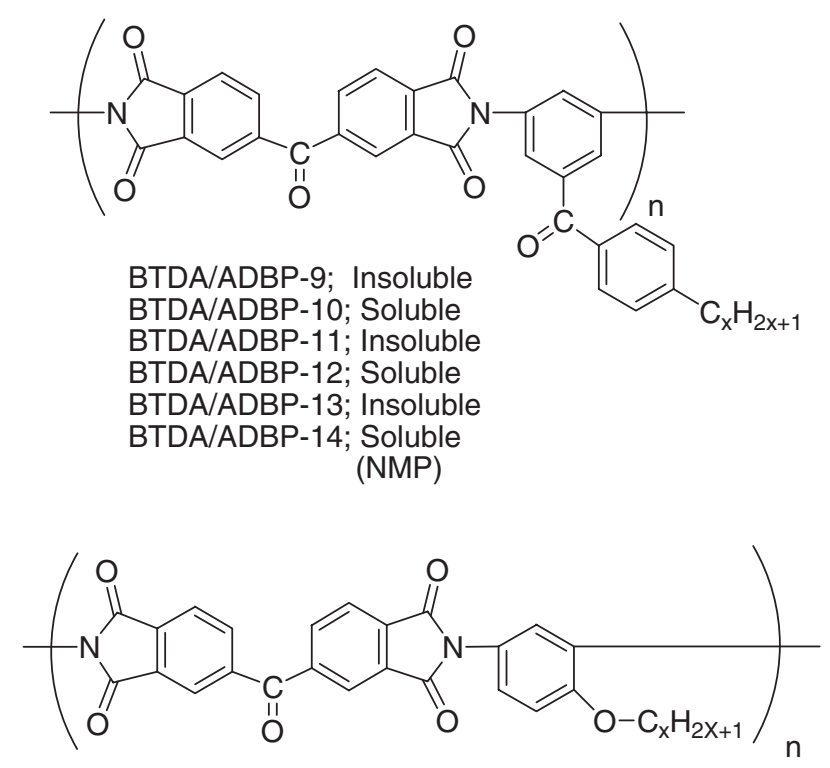

BTDA/AODB-10; Soluble BTDA/AODB-11; Soluble BTDA/AODB-12; Soluble BTDA/AODB-13; Soluble BTDA/AODB-14; Soluble (NMP)

Scheme 1. Soluble polyimides based on alkyldiaminobenzophenone (ADBP) and alkyloxydiaminobenzene (AODB).

tion of a novel series of soluble polyimides based on diaminobenzoic acid alkylester (DBAE) having longchain linear alkyl groups with $8-14$ carbon atoms. Although polyimides based on DBAE having 8, 12, or 18 carbon atom have already been reported, ${ }^{32}$ the structure-properties relationships of polymers were not investigated thoroughly. In this paper, the factors affecting the solubility of the final polymers, such as the alkyl chain length of DBAE, the use of branched alkyl chains and the copolymerization effects using the conventional aromatic diamine 4,4'-diaminodiphenylether (DDE) or 4,4'-diaminodiphenylmethane (DDM) are described, and the results are compared with the ones based on ADBP and AODB. The attaching of alkyl side chains to polyimides has recently been used to increase pretilt angles generated by the polyimides in alignment layer applications of liquid crystal displays (LCDs) and, thus, these studies are of great practical importance. ${ }^{33-39}$

\section{EXPERIMENTAL}

\section{Materials}

3,5-Dinitrobenzoyl chloride and aliphatic alcohol having long-chain alkyl groups were purchased from Tokyo Chemical Industry Co., Ltd. (TCI) and used as received, as were potassium carbonate and 10\% $\mathrm{Pd} / \mathrm{C}$, which were purchased from Wako Pure Chemical Industries, Ltd (Wako). 3,3' ${ }^{\prime}$ 4,4'-Benzophenone tetracarboxylic dianhydride (BTDA), 4,4'-diaminodiphenylether (DDE), and 4,4'-diaminodiphenylmethane (DDM) were purchased from Tokyo Chemical Industry Co., Ltd. (TCI) and purified by following method; BTDA, mp $228.3{ }^{\circ} \mathrm{C}$, recrystallized from acetic anhydride; DDE, mp $193.6^{\circ} \mathrm{C}$, recrystallized from ethanol; DDM, mp $95.4^{\circ} \mathrm{C}$, recrystallized from ethanol. $N$-Methyl-2-pyrrolidone (NMP) (Mitsubishi Chemicals) was distilled under reduced pressure from $4 \AA$ molecular sieves. Reagent grade $N, N$-dimethylformamide (DMF), $N, N$-dimethylacetamide (DMAc), dimethyl sulfoxide (DMSO), Dimethyl sulfoxide- $d_{6}$ (DMSO- $\left.d_{6}\right)$, 1,3-dimethyl-2-imidazolidinone (DMI), $m$-cresol, tetrahydrofuran (THF), dichloromethane, sulfuric acid, acetic anhydride and pyridine were purchased from Wako Pure Chemical Industries, Ltd. and used as received. Other conventional reagents were purchased from TCI or Wako and used as received.

\section{Measurements}

The inherent viscosities of all polymers were measured using Cannon Fenske viscometers at a concentration of $0.5 \mathrm{~g} / \mathrm{dL}$ in NMP at $30^{\circ} \mathrm{C}$. Differential scanning calorimeter (DSC) traces were measured on a Shimadzu DSC-60 under nitrogen at a heating rate of $10^{\circ} \mathrm{C} / \mathrm{min}$ and glass transition temperatures $\left(T_{\mathrm{g}}\right)$ were read at the onset of the heat capacity jump $\left(T_{\mathrm{ig}}\right)$ from the second heating scan followed by JIS K-7121. Thermogravimetric analysis (TGA) was performed on a Shimadzu TGA-50 in air or under nitrogen at a heating rate of $10^{\circ} \mathrm{C} / \mathrm{min} .{ }^{1} \mathrm{H}$ NMR spectra were measured on a JEOL JNM-AL400 FT NMR in $\mathrm{CDCl}_{3}$ or dimethyl sulfoxide- $d_{6}$ with tetramethylsilane (TMS) as an internal reference. IR spectra were measured on a JASCO IR Report-100 spectrophotometer.

\section{Synthesis of 2,6-Dimethylheptane-4-yl 3,5-dinitroben- zoate}

2,6-Dimethyl-4-heptanol $(7.5 \mathrm{~g}, 52 \mathrm{mmol})$ and triethylamine $(6.9 \mathrm{~g}, 68 \mathrm{mmol})$ were dissolved in $80 \mathrm{~mL}$ of THF. After 3,5-dinitrobenzoyl chloride $(12.0 \mathrm{~g}$, $52 \mathrm{mmol}$ ) in $80 \mathrm{~mL}$ of THF was added dropwise, the reaction mixture was stirred at $\mathrm{r}$. $\mathrm{t}$ for $3 \mathrm{~h}$. The reaction mixture was then poured onto a large amount of ice water. Ethyl acetate was added to dissolve the precipitate and the organic layer was separated and washed sequentially with $\mathrm{H}_{2} \mathrm{O}, 10 \%$ aqueous $\mathrm{NaOH}$, and $\mathrm{H}_{2} \mathrm{O}$. Subsequently, it was dried over anhydrous sodium sulfate, filtered and the ethyl acetate was evaporated. The obtained solid was recrystallized from ethanol with charcoal to give $11.1 \mathrm{~g}$ ( $33 \mathrm{mmol}, 63 \%$ yield) of white crystals. mp $47.5^{\circ} \mathrm{C} ;{ }^{1} \mathrm{H}$ NMR $\left(\mathrm{CDCl}_{3}\right): \delta$ $0.96\left(\mathrm{~m}, 12 \mathrm{H}, \mathrm{CH}_{3}\right), 1.47\left(\mathrm{~m}, 2 \mathrm{H},-\mathrm{CH}-\left(\mathrm{CH}_{3}\right)_{2}\right), 1.69$ $\left(\mathrm{m}, 4 \mathrm{H},-\mathrm{COO}-\mathrm{CH}-\left(\mathrm{CH}_{2}-\right)_{2}\right), 5.43(\mathrm{~m}, 1 \mathrm{H},-\mathrm{COO}-$ $\mathrm{CH}-), 8.58$ (d, 1H, 4-ArH, $J=3.96 \mathrm{~Hz}), 9.15(\mathrm{~d}, 2 \mathrm{H}$, 
2,6-ArH, $J=2.0 \mathrm{~Hz}), 9.22(\mathrm{t}, 1 \mathrm{H}, 4-\mathrm{ArH}, J=2.0$ $\mathrm{Hz})$; IR $(\mathrm{KBr}): 1720(\mathrm{C}=\mathrm{O}), 1540,1340\left(\mathrm{NO}_{2}\right) \mathrm{cm}^{-1}$. Anal. Calcd for $\mathrm{C}_{16} \mathrm{H}_{22} \mathrm{~N}_{2} \mathrm{O}_{5}$ : C, $56.80 \%$; H, 6.55\%; N, $8.28 \%$. Found: C, $56.72 \%$; H, 6.55\%; N, $8.33 \%$.

Other alkyl 3.5-dinitrobenzoates were synthesized according to the procedure descried above $(60-70 \%$ yield). Melting points (mp), ${ }^{1} \mathrm{H}$ NMR, IR and elemental analyses are as follows. In case of known compounds, only melting points are listed.

\section{n-Octyl 3,5-dinitrobenzoate}

mp $57.9^{\circ} \mathrm{C}$ (literature ${ }^{32} \mathrm{mp} 59^{\circ} \mathrm{C}$ )

\section{n-Nonyl 3,5-dinitrobenzoate}

mp 47.2 ${ }^{\circ} \mathrm{C} ;{ }^{1} \mathrm{H} \mathrm{NMR}\left(\mathrm{CDCl}_{3}\right): \delta 0.87\left(\mathrm{t}, 3 \mathrm{H}, \mathrm{CH}_{3}\right.$, $J=6.8 \mathrm{~Hz}), 1.30\left(\mathrm{~m}, 12 \mathrm{H},-\left(\mathrm{CH}_{2}\right)_{6} \mathrm{CH}_{3}\right), 1.84(\mathrm{~m}$, $2 \mathrm{H},-\mathrm{COOCH}_{2} \mathrm{CH}_{2}-$ ), 4.54 (t, $2 \mathrm{H},-\mathrm{COOCH}_{2} \mathrm{CH}_{2}-$, $J=6.8 \mathrm{~Hz}), 9.15(\mathrm{~d}, 2 \mathrm{H}, 2,6-\mathrm{ArH}, J=2.0 \mathrm{~Hz}), 9.22$ (t, $1 \mathrm{H}, 4-\mathrm{ArH}, J=2.0 \mathrm{~Hz})$; IR $(\mathrm{KBr}): 1720(\mathrm{C}=\mathrm{O})$, 1540, $1340\left(\mathrm{NO}_{2}\right) \mathrm{cm}^{-1}$. Anal. Calcd for $\mathrm{C}_{16} \mathrm{H}_{22}-$ $\mathrm{N}_{2} \mathrm{O}_{6}: \mathrm{C}, 56.80 \% ; \mathrm{H}, 6.55 \%$;, $8.28 \%$. Found: $\mathrm{C}$, $56.93 \% ; \mathrm{H}, 6.58 \%$; N, 8.34\%.

\section{n-Decyl 3,5-dinitrobenzoate}

mp $44.6{ }^{\circ} \mathrm{C} ;{ }^{1} \mathrm{H} \mathrm{NMR}\left(\mathrm{CDCl}_{3}\right): \delta 0.88\left(\mathrm{t}, 3 \mathrm{H}, \mathrm{CH}_{3}\right.$, $J=6.8 \mathrm{~Hz}), 1.27\left(\mathrm{~m}, 14 \mathrm{H},-\left(\mathrm{CH}_{2}\right)_{7} \mathrm{CH}_{3}\right), 1.84(\mathrm{~m}$, $\left.2 \mathrm{H},-\mathrm{COOCH}_{2} \mathrm{CH}_{2}-\right), 4.52\left(\mathrm{t}, 2 \mathrm{H},-\mathrm{COOCH}_{2} \mathrm{CH}_{2}-\right.$, $J=6.8 \mathrm{~Hz}), 9.15(\mathrm{~d}, 2 \mathrm{H}, 2,6-\mathrm{ArH}, J=2.0 \mathrm{~Hz}), 9.22$ (t, $1 \mathrm{H}, 4-\mathrm{ArH}, J=2.0 \mathrm{~Hz})$; IR $(\mathrm{KBr}): 1720(\mathrm{C}=\mathrm{O})$, 1540, $1340\left(\mathrm{NO}_{2}\right) \mathrm{cm}^{-1}$. Anal. Calcd for $\mathrm{C}_{17} \mathrm{H}_{24}{ }^{-}$ $\mathrm{N}_{2} \mathrm{O}_{6}$ : C, 57.94\%; H, 6.87\%; N, 7.95\%. Found: C, $58.07 \% ; \mathrm{H}, 6.87 \%$;, $8.00 \%$.

\section{n-Undecyl 3,5-dinitrobenzoate}

mp 51.9 ${ }^{\circ} \mathrm{C} ;{ }^{1} \mathrm{H}$ NMR $\left(\mathrm{CDCl}_{3}\right): \delta 0.88\left(\mathrm{t}, 3 \mathrm{H}, \mathrm{CH}_{3}\right.$, $J=6.8 \mathrm{~Hz}), 1.27\left(\mathrm{~m}, 16 \mathrm{H},-\left(\mathrm{CH}_{2}\right)_{8} \mathrm{CH}_{3}\right), 1.83(\mathrm{~m}$, $\left.2 \mathrm{H},-\mathrm{COOCH}_{2} \mathrm{CH}_{2}-\right), 4.54\left(\mathrm{t}, 2 \mathrm{H},-\mathrm{COOCH}_{2} \mathrm{CH}_{2}-\right.$, $J=6.8 \mathrm{~Hz}), 9.15(\mathrm{~d}, 2 \mathrm{H}, 2,6-\mathrm{ArH}, J=2.0 \mathrm{~Hz}), 9.22$ (t, $1 \mathrm{H}, 4-\mathrm{ArH}, J=2.0 \mathrm{~Hz})$; IR $(\mathrm{KBr}): 1720(\mathrm{C}=\mathrm{O})$, 1540, $1340\left(\mathrm{NO}_{2}\right) \mathrm{cm}^{-1}$. Anal. Calcd for $\mathrm{C}_{18} \mathrm{H}_{26^{-}}$ $\mathrm{N}_{2} \mathrm{O}_{6}: \mathrm{C}, 59.00 \% ; \mathrm{H}, 7.15 \% ; \mathrm{N}, 7.65 \%$. Found: C, $59.05 \%$; H, 7.15\%; N, 7.66\%.

\section{n-Dodecyl 3,5-dinitrobenzoate}

mp $55.8^{\circ} \mathrm{C}$ (literature ${ }^{32} \mathrm{mp} 57-59^{\circ} \mathrm{C}$ )

\section{n-Tridecyl 3,5-dinitrobenzoate}

mp 58.4 ${ }^{\circ} \mathrm{C} ;{ }^{1} \mathrm{H}$ NMR $\left(\mathrm{CDCl}_{3}\right): \delta 0.88\left(\mathrm{t}, 3 \mathrm{H}, \mathrm{CH}_{3}\right.$, $J=6.8 \mathrm{~Hz}), 1.27\left(\mathrm{~m}, 20 \mathrm{H},-\left(\mathrm{CH}_{2}\right)_{10} \mathrm{CH}_{3}\right), 1.83(\mathrm{~m}$, $2 \mathrm{H},-\mathrm{COOCH}_{2} \mathrm{CH}_{2}-$ ), 4.54 (t, $2 \mathrm{H},-\mathrm{COOCH}_{2} \mathrm{CH}_{2}-$, $J=6.8 \mathrm{~Hz}), 9.15(\mathrm{~d}, 2 \mathrm{H}, 2,6-\mathrm{ArH}, J=2.0 \mathrm{~Hz}), 9.22$ (t, $1 \mathrm{H}, 4-\mathrm{ArH}, J=2.0 \mathrm{~Hz})$; IR $(\mathrm{KBr}): 1720(\mathrm{C}=\mathrm{O})$, 1540, $1340\left(\mathrm{NO}_{2}\right) \mathrm{cm}^{-1}$. Anal. Calcd for $\mathrm{C}_{20} \mathrm{H}_{30^{-}}$ $\mathrm{N}_{2} \mathrm{O}_{6}: \mathrm{C}, 60.90 \% ; \mathrm{H}, 7.67 \% ; \mathrm{N}, 7.10 \%$. Found: C, $60.75 \% ; \mathrm{H}, 7.76 \%$;, $7.23 \%$.

\section{n-Tetradecyl 3,5-dinitrobenzoate}

mp $58.8{ }^{\circ} \mathrm{C} ;{ }^{1} \mathrm{H} \mathrm{NMR}\left(\mathrm{CDCl}_{3}\right): \delta 0.87\left(\mathrm{t}, 3 \mathrm{H}, \mathrm{CH}_{3}\right.$, $J=6.8 \mathrm{~Hz}), 1.26\left(\mathrm{~m}, 22 \mathrm{H},-\left(\mathrm{CH}_{2}\right)_{11} \mathrm{CH}_{3}\right), 1.83(\mathrm{~m}$, $2 \mathrm{H},-\mathrm{COOCH}_{2} \mathrm{CH}_{2}-$ ), 4.44 (t, $2 \mathrm{H},-\mathrm{COOCH}_{2} \mathrm{CH}_{2}-$, $J=6.8 \mathrm{~Hz}), 9.15(\mathrm{~d}, 2 \mathrm{H}, 2,6-\mathrm{ArH}, J=2.0 \mathrm{~Hz}), 9.22$ (t, $1 \mathrm{H}, 4-\mathrm{ArH}, J=2.0 \mathrm{~Hz})$; IR $(\mathrm{KBr}): 1720(\mathrm{C}=\mathrm{O})$, 1540, $1340\left(\mathrm{NO}_{2}\right) \mathrm{cm}^{-1}$. Anal. Calcd for $\mathrm{C}_{21} \mathrm{H}_{32}-$ $\mathrm{N}_{2} \mathrm{O}_{6}: \mathrm{C}, 61.75 \% ; \mathrm{H}, 7.90 \%$; N $6.86 \%$. Found: C, $61.72 \% ; \mathrm{H}, 7.88 \%$; N $6.81 \%$.

\section{Nonan-5-yl 3,5-dinitrobenzoate}

mp $52.0{ }^{\circ} \mathrm{C} ;{ }^{1} \mathrm{H} \mathrm{NMR}\left(\mathrm{CDCl}_{3}\right): \delta 0.91\left(\mathrm{t}, 6 \mathrm{H}, \mathrm{CH}_{3}\right.$, $J=7 \mathrm{~Hz}), 1.35\left(\mathrm{~m}, 8 \mathrm{H},-\left(\mathrm{CH}_{2}\right)_{2} \mathrm{CH}_{3}\right), 1.74(\mathrm{~m}, 4 \mathrm{H}$, - $\left.\mathrm{COO}-\left(\mathrm{CHCH}_{2}-\right)_{2}\right), 5.24$ (m, 1H, -COO-CH-), 9.15 $(\mathrm{d}, 2 \mathrm{H}, 2,6-\mathrm{ArH}, J=2.2 \mathrm{~Hz}), 9.22(\mathrm{t}, 1 \mathrm{H}, 4-\mathrm{ArH}, J=$ $2.2 \mathrm{~Hz})$; IR $(\mathrm{KBr}): 1710(\mathrm{C}=\mathrm{O}), 1540,1340\left(\mathrm{NO}_{2}\right)$ $\mathrm{cm}^{-1}$. Anal. Calcd for $\mathrm{C}_{16} \mathrm{H}_{22} \mathrm{~N}_{2} \mathrm{O}_{5}: \mathrm{C}, 56.80 \% ; \mathrm{H}$, $6.55 \%$; N, 8.28\%. Found: C, 56.72\%; H, 6.65\%; N, $8.27 \%$.

Synthesis of 2,6-Dimethylheptane-4-yl 3,5-diaminobenzoate (DBAE-9-branch-B)

To a solution of 2,6-dimethylheptane-4-yl 3,5-dinitrobenzoate $(9.5 \mathrm{~g}, 28 \mathrm{mmmol})$ in $200 \mathrm{~mL}$ of ethanol heated at $50{ }^{\circ} \mathrm{C}$ were sequentially added $0.20 \mathrm{~g}$ of $10 \%$ $\mathrm{Pd} / \mathrm{C}$ by potion, $26 \mathrm{~mL}$ of hydrazine monohydrate using dropping funnel, and $0.20 \mathrm{~g}$ of $10 \% \mathrm{Pd} / \mathrm{C}$ by potion. After this reaction mixture was refluxed for $12 \mathrm{~h}$, the solution was filtered and the solvent was evaporated. The crude diamine was recrystallized from an ethanol/water mixture with charcoal to give $6.6 \mathrm{~g}\left(85 \%\right.$ yield) of a pale brown powder. $\mathrm{mp} 98.9^{\circ} \mathrm{C}$; ${ }^{1} \mathrm{H}$ NMR $\left(\mathrm{CDCl}_{3}\right): \delta 0.92\left(\mathrm{~m}, 12 \mathrm{H}, \mathrm{CH}_{3}\right), 1.39(\mathrm{~m}$, $\left.2 \mathrm{H},-\mathrm{CH}-\left(\mathrm{CH}_{3}\right)_{2}\right), 1.63$ (m, 4H, $\left.-\mathrm{COO}-\mathrm{CH}-\left(\mathrm{CH}_{2}-\right)_{2}\right)$, $3.52\left(\mathrm{~s}, 4 \mathrm{H},-\mathrm{NH}_{2}\right), 5.27(\mathrm{~m}, 1 \mathrm{H},-\mathrm{COO}-\mathrm{CH}-), 6.18$ $(\mathrm{t}, 1 \mathrm{H}, 4-\mathrm{ArH}, J=2.2 \mathrm{~Hz}), 6.78(\mathrm{~d}, 2 \mathrm{H}, 2,6-\mathrm{ArH}, J=$ $2.2 \mathrm{~Hz})$; IR (KBr): $3350\left(\mathrm{NH}_{2}\right), 1720(\mathrm{C}=\mathrm{O}) \mathrm{cm}^{-1}$. Anal. Calcd for $\mathrm{C}_{16} \mathrm{H}_{26} \mathrm{~N}_{2} \mathrm{O}_{2}$ : C, 69.03\%; $\mathrm{H}, 9.41 \%$; N, $10.06 \%$. Found: C, $69.05 \%$; H, $9.42 \%$; N, $10.07 \%$.

Other alkyl 3,5-diaminobenzoates were synthesized according to the procedure descried above $(63-84 \%$ yield). Melting points (mp), ${ }^{1} \mathrm{H}$ NMR, IR and elemental analyses are as follows. In case of known compounds, only melting points are listed.

\section{n-Octyl 3,5-diaminobenzoate}

mp $50.1^{\circ} \mathrm{C}$ (literature ${ }^{32} \mathrm{mp} 32-33.5^{\circ} \mathrm{C}$ )

\section{n-Nonyl 3,5-diaminobenzoate}

mp $33.2{ }^{\circ} \mathrm{C} ;{ }^{1} \mathrm{H} \mathrm{NMR}\left(\mathrm{CDCl}_{3}\right): \delta 0.88\left(\mathrm{t}, 3 \mathrm{H}, \mathrm{CH}_{3}\right.$, $J=6.80 \mathrm{~Hz}), 1.28\left(\mathrm{~m}, 12 \mathrm{H},-\left(\mathrm{CH}_{2}\right)_{6}-\mathrm{CH}_{3}\right), 1.71(\mathrm{~m}$, $\left.2 \mathrm{H},-\mathrm{COOCH}_{2} \mathrm{CH}_{2}-\right), 3.68\left(\mathrm{~s}, 4 \mathrm{H},-\mathrm{NH}_{2}\right), 4.25(\mathrm{t}, 2 \mathrm{H}$, $\left.-\mathrm{COOCH}_{2} \mathrm{CH}_{2}-, J=6.80 \mathrm{~Hz}\right), 6.18(\mathrm{t}, 1 \mathrm{H}, 4-\mathrm{ArH}$, $J=2.0 \mathrm{~Hz}), 6.78(\mathrm{~d}, 2 \mathrm{H}, 2,6-\mathrm{ArH}, J=2.0 \mathrm{~Hz}) ; \mathrm{IR}$ (KBr): $3350\left(\mathrm{NH}_{2}\right), 1720(\mathrm{C}=\mathrm{O}) \mathrm{cm}^{-1}$. Anal. Calcd 
for $\mathrm{C}_{16} \mathrm{H}_{26} \mathrm{~N}_{2} \mathrm{O}_{2}$ : C, 69.03\%; H, 9.41\%; N, $10.06 \%$. Found: C, 68.81\%; H, 9.24\%; N, 9.89\%.

\section{n-Decyl 3,5-diaminobenzoate}

mp $50.1{ }^{\circ} \mathrm{C} ;{ }^{1} \mathrm{H} \mathrm{NMR}\left(\mathrm{CDCl}_{3}\right): \delta 0.88\left(\mathrm{t}, 3 \mathrm{H}, \mathrm{CH}_{3}\right.$, $J=6.80 \mathrm{~Hz}), 1.27\left(\mathrm{~m}, 14 \mathrm{H},-\left(\mathrm{CH}_{2}\right)_{7}-\mathrm{CH}_{3}\right), 1.71(\mathrm{~m}$, $\left.2 \mathrm{H},-\mathrm{COOCH}_{2} \mathrm{CH}_{2}-\right), 3.64\left(\mathrm{~s}, 4 \mathrm{H},-\mathrm{NH}_{2}\right), 4.25(\mathrm{t}, 2 \mathrm{H}$, $\left.-\mathrm{COOCH}_{2} \mathrm{CH}_{2}-, J=6.80 \mathrm{~Hz}\right), 6.18(\mathrm{t}, 1 \mathrm{H}, 4-\mathrm{ArH}$, $J=2.0 \mathrm{~Hz}), 6.78(\mathrm{~d}, 2 \mathrm{H}, 2,6-\mathrm{ArH}, J=2.0 \mathrm{~Hz}) ; \mathrm{IR}$ $(\mathrm{KBr}): 3350\left(\mathrm{NH}_{2}\right), 1720(\mathrm{C}=\mathrm{O}) \mathrm{cm}^{-1}$. Anal. Calcd for $\mathrm{C}_{17} \mathrm{H}_{28} \mathrm{~N}_{2} \mathrm{O}_{2}$ : C, 69.83\%; H, 9.65\%; N, 9.58\%. Found: C, 69.74\%; H, 9.61\%; N, 9.59\%.

\section{n-Undecyl 3,5-diaminobenzoate}

mp 53.9 ${ }^{\circ} \mathrm{C} ;{ }^{1} \mathrm{H} \mathrm{NMR}\left(\mathrm{CDCl}_{3}\right): \delta 0.88\left(\mathrm{t}, 3 \mathrm{H}, \mathrm{CH}_{3}\right.$, $J=6.80 \mathrm{~Hz}), 1.27\left(\mathrm{~m}, 16 \mathrm{H},-\left(\mathrm{CH}_{2}\right)_{8}-\mathrm{CH}_{3}\right), 1.71(\mathrm{~m}$, $\left.2 \mathrm{H},-\mathrm{COOCH}_{2} \mathrm{CH}_{2}-\right), 3.66$ (s, $\left.4 \mathrm{H},-\mathrm{NH}_{2}\right), 4.25$ (t, $2 \mathrm{H}$, $\left.-\mathrm{COOCH}_{2} \mathrm{CH}_{2}-, J=6.80 \mathrm{~Hz}\right), 6.19(\mathrm{t}, 1 \mathrm{H}, 4-\mathrm{ArH}$, $J=2.0 \mathrm{~Hz}), 6.78(\mathrm{~d}, 2 \mathrm{H}, 2,6-\mathrm{ArH}, J=2.0 \mathrm{~Hz})$; IR $(\mathrm{KBr}): 3350\left(\mathrm{NH}_{2}\right), 1720(\mathrm{C}=\mathrm{O}) \mathrm{cm}^{-1}$. Anal. Calcd for $\mathrm{C}_{18} \mathrm{H}_{30} \mathrm{~N}_{2} \mathrm{O}_{2}$ : C, 70.55\%; H, 9.87\%; N, 9.14\%. Found: C, 70.45\%; H, 9.82\%; N, 9.08\%.

\section{n-Dodecyl 3,5-diaminobenzoate}

mp $80.2^{\circ} \mathrm{C}$ (literature ${ }^{32} \mathrm{mp} 68-70^{\circ} \mathrm{C}$ )

\section{n-Tridecyl 3,5-diaminobenzoate}

mp 59.4 ${ }^{\circ} \mathrm{C} ;{ }^{1} \mathrm{H} \mathrm{NMR}\left(\mathrm{CDCl}_{3}\right): \delta 0.88\left(\mathrm{t}, 3 \mathrm{H}, \mathrm{CH}_{3}\right.$, $J=6.80 \mathrm{~Hz}), 1.26\left(\mathrm{~m}, 20 \mathrm{H},-\left(\mathrm{CH}_{2}\right)_{10}-\mathrm{CH}_{3}\right), 1.71(\mathrm{~m}$, $\left.2 \mathrm{H},-\mathrm{COOCH}_{2} \mathrm{CH}_{2}-\right), 3.69\left(\mathrm{~s}, 4 \mathrm{H},-\mathrm{NH}_{2}\right), 4.25(\mathrm{t}, 2 \mathrm{H}$, $\left.-\mathrm{COOCH}_{2} \mathrm{CH}_{2}-, J=6.80 \mathrm{~Hz}\right), 6.19(\mathrm{t}, 1 \mathrm{H}, 4-\mathrm{ArH}$, $J=2.0 \mathrm{~Hz}), 6.78(\mathrm{~d}, 2 \mathrm{H}, 2,6-\mathrm{ArH}, J=2.0 \mathrm{~Hz}) ; \mathrm{IR}$ $(\mathrm{KBr}): 3350\left(\mathrm{NH}_{2}\right), 1720(\mathrm{C}=\mathrm{O}) \mathrm{cm}^{-1}$. Anal. Calcd for $\mathrm{C}_{20} \mathrm{H}_{34} \mathrm{~N}_{2} \mathrm{O}_{2}$ : C, $71.81 \%$; $\mathrm{H}, 10.25 \%$; N, $8.37 \%$. Found: C, $71.71 \%$; H, $10.20 \%$; N, $8.37 \%$.

\section{n-Tetradecyl 3,5-diaminobenzoate}

$\mathrm{mp} 64.2{ }^{\circ} \mathrm{C} ;{ }^{1} \mathrm{H} \mathrm{NMR}\left(\mathrm{CDCl}_{3}\right): \delta 0.88\left(\mathrm{t}, 3 \mathrm{H}, \mathrm{CH}_{3}\right.$, $J=6.80 \mathrm{~Hz}), 1.26\left(\mathrm{~m}, 22 \mathrm{H},-\left(\mathrm{CH}_{2}\right)_{11}-\mathrm{CH}_{3}\right), 1.71(\mathrm{~m}$, $\left.2 \mathrm{H},-\mathrm{COOCH}_{2} \mathrm{CH}_{2}-\right), 3.65\left(\mathrm{~s}, 4 \mathrm{H},-\mathrm{NH}_{2}\right), 4.25(\mathrm{t}, 2 \mathrm{H}$, $\left.-\mathrm{COOCH}_{2} \mathrm{CH}_{2}-, J=6.80 \mathrm{~Hz}\right), 6.18(\mathrm{t}, 1 \mathrm{H}, 4-\mathrm{ArH}$, $J=2.0 \mathrm{~Hz}), 6.78$ (d, 2H, 2,6-ArH, $J=2.0 \mathrm{~Hz}$ ); IR $(\mathrm{KBr}): 3350\left(\mathrm{NH}_{2}\right), 1720(\mathrm{C}=\mathrm{O}) \mathrm{cm}^{-1}$. Anal. Calcd for $\mathrm{C}_{21} \mathrm{H}_{36} \mathrm{~N}_{2} \mathrm{O}_{2}$ : C, $72.37 \% ; \mathrm{H}, 10.41 \%$; N, $8.04 \%$. Found: C, 72.40\%; H, 10.44\%; N, 7.93\%.

Nonan-5-yl 3,5-diaminobenzoate (DBAE-9-branch-A) Liquid; ${ }^{1} \mathrm{H}$ NMR $\left(\mathrm{CDCl}_{3}\right): \delta 0.88\left(\mathrm{t}, 6 \mathrm{H}, \mathrm{CH}_{3}\right.$, $J=7 \mathrm{~Hz}), 1.33\left(\mathrm{~m}, 8 \mathrm{H},-\left(\mathrm{CH}_{2}\right)_{2} \mathrm{CH}_{3}\right), 1.62(\mathrm{~m}, 4 \mathrm{H}$, $\left.-\mathrm{COO}-\left(\mathrm{CHCH}_{2}-\right)_{2}\right), 3.51$ (s, $\left.4 \mathrm{H},-\mathrm{NH}_{2}\right), 5.07(\mathrm{~m}, 1 \mathrm{H}$, -COO-CH-), 6.18 (t, 1H, 4-ArH, $J=2.0 \mathrm{~Hz}$ ), 6.78 (d, $2 \mathrm{H}, 2,6-\mathrm{ArH}, J=2.0 \mathrm{~Hz})$; IR $(\mathrm{KBr}): 3350\left(\mathrm{NH}_{2}\right)$, $1720(\mathrm{C}=\mathrm{O}) \mathrm{cm}^{-1}$. Anal. Calcd for $\mathrm{C}_{16} \mathrm{H}_{26} \mathrm{~N}_{2} \mathrm{O}_{2}$ : C, 69.03\%; H, 9.41\%; N, 10.06\%. Found: C, 68.83\%; $\mathrm{H}, 9.33 \%$; N, $9.81 \%$.
Preparation of Poly(amic acids) and Polyimides

The preparation of poly(amic acids) and polyimides was carried out by the method previously reported. $^{25-29}$ The imidization reaction of the poly(amic acids) was monitored by ${ }^{1} \mathrm{H}$ NMR technique and the conversion from poly(amic acids) to polyimides were found to be $100 \%$. Copoly (amic acids) and copolyimides using DDE or DDM as a diamine co-momomer were synthesized in the same manner. An equimolar amount of BTDA was used with respect to the total amount of diamines and all monomers were added at the beginning of reaction. Diamine compositions of obtained copolyimides were confirmed by ${ }^{1} \mathrm{H}$ NMR and detailed description are given in the next section. ${ }^{1} \mathrm{H}$ NMR spectra, IR Spectra and results of elemental analysis of representative polyimides are as follows.

\section{BTDA/DBAE-9-branch-B (100/100 mol \%)}

${ }^{1} \mathrm{H}$ NMR (DMSO- $\left.d_{6}\right): \delta 0.89\left(\mathrm{~m}, 12 \mathrm{H}, \mathrm{CH}_{3}\right), 1.43$ $\left(\mathrm{m}, 2 \mathrm{H},-\mathrm{CH}-\left(\mathrm{CH}_{3}\right)_{2}\right), 1.63(\mathrm{~m}, 4 \mathrm{H},-\mathrm{COO}-\mathrm{CH}-$ $\left.\left(\mathrm{CH}_{2}-\right)_{2}\right), 5.30(\mathrm{~m}, 1 \mathrm{H},-\mathrm{COO}-\mathrm{CH}-), 8.15-8.30(\mathrm{~m}$, 9H, ArH) ppm; IR (KBr): 1715 and $1770(\mathrm{C}=\mathrm{O})$, $1360(\mathrm{C}-\mathrm{N}) \mathrm{cm}^{-1}$. Anal. Calcd for $\mathrm{C}_{33} \mathrm{H}_{30} \mathrm{~N}_{2} \mathrm{O}_{6}$ : C, 71.99\%; H, 5.49\%; N, 5.09\%. Found C, 70.78\%; H, $5.31 \% ; \mathrm{N}, 5.39 \%$.

\section{RESULTS AND DISCUSSION}

\section{Monomer Synthesis}

The functional diamines containing long-chain alkyl groups, diaminobenzoic acid alkylester (DBAE$\mathrm{X} ; \mathrm{X}$, alkyl chain length), were prepared in two steps using 3,5-dinitrobenzoyl chloride as a starting material. The synthetic route is illustrated in Scheme 2. The esterification reaction using 3,5-dinitrobenzoyl chloride and aliphatic alcohols having long-chain alkyl groups catalyzed by triethyl amine in THF gave alkyl 3,5-dinitrobenzoate in satisfactory yields $(60-70 \%)$. The reduction of alkyl 3,5-dinitrobenzoate were performed by catalytic hydrogenation using $\mathrm{Pd} / \mathrm{C}$ as a catalyst and hydrazine hydrate/ethanol as a hydrogen generator. Although the medium pressure hydrogenerator was used in our laboratory to complete the reduction of dinitro precursors such as 1-alkyloxy2,4-dinitrobenzene, ${ }^{29}$ the relatively mild hydrogenation using hydrazine hydrate/ethanol system was seem to be preferable in the case of alkyl 3,5-dinitrobenzoate, because the scissions of ester linkage were sometime recognized in the use of medium pressure hydrogenerator.

\section{Polymer Synthesis}

The synthetic route of the polyimides and copolyimides based on BTDA, DBAE-X, DDE or DDM is 
<smiles>O=C(Cl)c1cc([N+](=O)[O-])cc([N+](=O)[O-])c1</smiles>

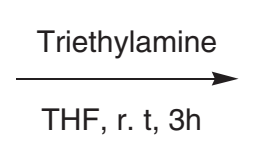

THF, r. t, 3h<smiles>O=C(O)c1cc([N+](=O)[O-])cc([N+](=O)[O-])c1</smiles><smiles>[R]OC(=O)c1cc(N)cc(N)c1</smiles>

DBAE-8-14 $\left(\mathrm{R}=\mathrm{n}-\mathrm{C}_{8} \mathrm{H}_{17} \sim \mathrm{n}-\mathrm{C}_{14} \mathrm{H}_{29}\right)$

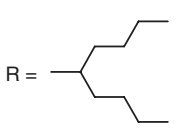

DBAE-9-branch-A DBAE-9-branch-B

Scheme 2. Synthesis of diaminobenzoic acid alkylester (DBAE).

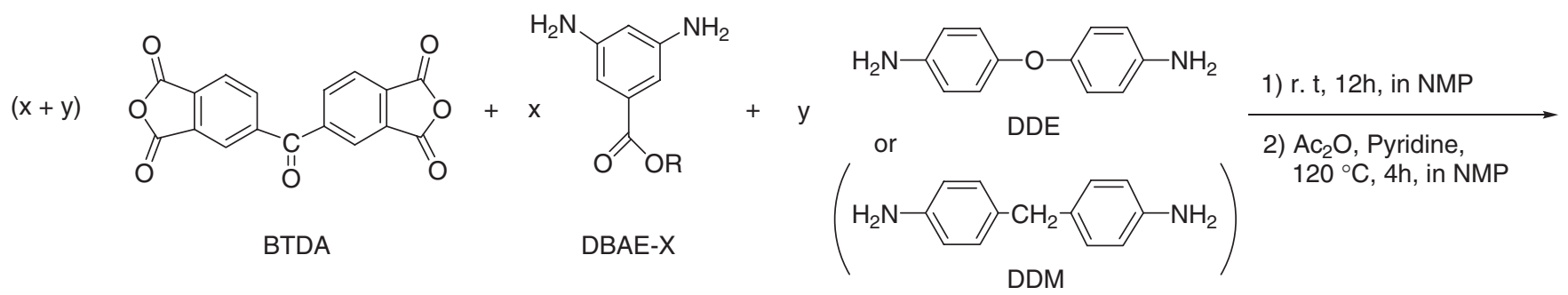<smiles>[R]OC(=O)c1cc(N2C(=O)c3ccc(C(=O)c4ccc5c(c4)C(=O)N(C)C5(C)C)cc3C2=O)cc(C(C)(C)C)c1</smiles>

DBAE-X: DBAE-8-14 $\left(\mathrm{R}=\mathrm{n}-\mathrm{C}_{8} \mathrm{H}_{17} \sim \mathrm{n}-\mathrm{C}_{14} \mathrm{H}_{29}\right)$
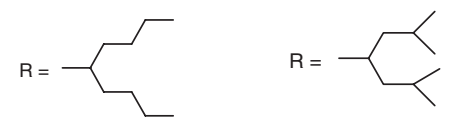

DBAE-9-branch-A DBAE-9-branch-B

Scheme 3. Synthesis of polyimides and copolyimides based on diaminobenzoic acid alkylester (DBAE).

illustrated in Scheme 3. Two step polymerization systems including poly(amic acids) synthesis and chemical imidization were performed. The poly(amic acids) were obtained by reacting the mixture of diamines with an equimolar amount of BTDA at room temperature for $12 \mathrm{~h}$ under a nitrogen atmosphere. The polyimides were obtained by chemical imidization at $120^{\circ} \mathrm{C}$ in the presence of pyridine as base catalyst and acetic anhydride as dehydrating agent. These are the optimized synthetic conditions previously developed for the synthesis of soluble polyimides in our laboratory. ${ }^{25-29}$ BTDA that is high reactive and common aromatic tetracarboxylic dianhydride was used as a dianhydride monomer, and DDE or DDM high reactive and common aromatic diamine was used as a diamine co-monomer.
Experimental results of homopolymerization and copolymerization based on BTDA/DBAE-X/DDE are summarized in Table I. Although all polyamic(acids) were soluble in NMP that is a polymerization solvent, however, all homopolyimide based on BTDA and DBAE-8-14 were insoluble in NMP. As it was previously reported that the copolymerization using conventional diamines such as DDE improve the solubility by randomizing effect, ${ }^{28,29}$ the incorporation of the second diamine monomer, DDE was examined. However, all copolyimide based on BTDA/DBAE-X/ DDE were insoluble although 25,50 , or $75 \mathrm{~mol} \%$ of DDE were incorporated. According to the common fact that branched alkyl chain often enhance the solubility of polymers in comparison with linear alkyl chains, DBAE having branched alkyl chains such as 
Table I. Polyimides and copolyimides based on BTDA/DBAE-X/DDE

\begin{tabular}{|c|c|c|c|c|c|c|c|c|c|}
\hline \multicolumn{2}{|c|}{ Diamine $^{\mathrm{a}}$} & \multirow{5}{*}{$\begin{array}{c}\frac{\text { Polyamic acid }}{} \\
\eta_{\text {inh }}{ }^{\mathrm{b}} \\
\mathrm{dLg}^{-1}\end{array}$} & \multicolumn{7}{|c|}{ Polyimide } \\
\hline & & & \multirow{4}{*}{$\begin{array}{l}\text { Solubility } \\
\text { in NMP }\end{array}$} & \multirow{4}{*}{$\frac{\eta_{\text {inh }^{\mathrm{b}}}}{\mathrm{dLg}^{-1}}$} & \multicolumn{2}{|c|}{ Diamine composition $^{\mathrm{c}}$} & \multirow{4}{*}{$\frac{T_{\mathrm{g}}^{\mathrm{d}}}{{ }^{\circ} \mathrm{C}}$} & \multirow{2}{*}{\multicolumn{2}{|c|}{$\begin{array}{c}10 \% \text { Weight loss } \\
\text { temperature }^{\mathrm{e}}\end{array}$}} \\
\hline DBAE-X & DDE & & & & DBAE-X & DDE & & & \\
\hline \multirow{2}{*}{\multicolumn{2}{|c|}{$\mathrm{mol} \%$}} & & & & & & & in Air & in $\mathrm{N}_{2}$ \\
\hline & & & & & & & & ${ }^{\circ} \mathrm{C}$ & ${ }^{\circ} \mathrm{C}$ \\
\hline \multicolumn{10}{|c|}{ DBAE-8-14 } \\
\hline 0 & 100 & 1.15 & insoluble & & & & & & \\
\hline 25 & 75 & $0.68-0.80$ & insoluble & & & & & & \\
\hline 50 & 50 & $0.57-0.65$ & insoluble & & & & & & \\
\hline 75 & 25 & $0.40-0.50$ & insoluble & & & & & & \\
\hline 100 & 0 & $0.39-0.43$ & insoluble & & & & & & \\
\hline \multicolumn{10}{|c|}{ DBAE-9-branch-A } \\
\hline 0 & 100 & 1.15 & insoluble & & & & & & \\
\hline 25 & 75 & 1.05 & insoluble & & & & & & \\
\hline 50 & 50 & 1.02 & soluble & 0.83 & & & 278 & 346 & 351 \\
\hline 75 & 25 & 1.13 & soluble & 0.82 & 76 & 24 & 280 & 340 & 333 \\
\hline 100 & 0 & 0.90 & soluble & 0.88 & & & 286 & 334 & 331 \\
\hline \multicolumn{10}{|c|}{ DBAE-9-branch-B } \\
\hline 0 & 100 & 1.15 & insoluble & & & & & & \\
\hline 25 & 75 & 1.02 & insoluble & & & & & & \\
\hline 50 & 50 & 0.99 & soluble & 0.93 & & & 292 & 348 & 340 \\
\hline 75 & 25 & 0.88 & soluble & 0.86 & 76 & 24 & 278 & 330 & 330 \\
\hline 100 & 0 & 0.89 & soluble & 0.84 & & & 296 & 323 & 327 \\
\hline
\end{tabular}

${ }^{\mathrm{a}}$ Equimolar amount of BTDA was used to the total amount of diamine. ${ }^{\mathrm{b}}$ Measured at $0.5 \mathrm{gdL}^{-1}$ in $\mathrm{NMP}$ at $30^{\circ} \mathrm{C}$. ${ }^{\mathrm{c}}$ Calculated from the intensity ratio of methine proton based on DBAE-9-branch-A(B) and aromatic protons based on DDE in ${ }^{1} \mathrm{H}$ NMR measurement. ${ }^{\mathrm{d}}$ Measured by DSC at a heating rate of $10^{\circ} \mathrm{C} / \mathrm{min}$ in $\mathrm{N}_{2}$ on second heating. ${ }^{\mathrm{e}}$ Measured by TGA at a heating rate of $10^{\circ} \mathrm{C} / \mathrm{min}$.

nonan-5-yl 3,5-diaminobenzoate (DBAE-9-branch-A) and 2,6-dimethylheptane-4-yl 3,5-diaminobenzoate (DBAE-9-branch-B) were introduced in these polyimides, and the homopolyimides based on BTDA/ DBAE-9-branch-A and BTDA/DBAE-9-branch-B, and copolyimide containing more than $50 \%$ of DBAE-9-branch-A or DBAE-9-branch-B were soluble in NMP. Thus, the effect of long chain alkyl groups for the enhancement of solubility were not recognized in the case of polyimides containing DBAE$8-14$, however, it was found that the introduction of branched alkyl chains enhance the solubility. Diamine compositions in the final copolyimides can be calculated from ${ }^{1} \mathrm{H}$ NMR measurement in DMSO- $d_{6}$ (Table I, Figures 1 and 2). Specifically, the diamine compositions of DBAE-9-branch-A/DDE (75\%/ $25 \%)$ and DBAE-9-branch-B/DDE (75\%/25\%) were calculated from the intensity ratio of methine proton based on DBAE-9-branch-A(B) and aromatic protons based on DDE, and in a fair agreement with the initial monomer compositions (Table I). In the case of DBAE-9-branch-A/DDE (50\%/50\%) and DBAE-9branch-B/DDE (50\%/50\%), the solubility in DMSO$d_{6}$ is poor and the accurate measurements were not achieved. Inherent viscosities $\left(\eta_{\text {inh }}\right)$, which are connected to the molecular weights of polymers, were sufficiently high in the range of $0.82-0.93 \mathrm{dLg}^{-1}$ for the copolyimide based on DBAE-9-branch-A and DBAE-9-branch-B, showing the good film formation ability.

Experimental results of homopolymerization and copolymerization based on BTDA/DBAE-X/DDM are summarized in Table II. As the solubility of above copolyimides using DDE as a diamine co-monomer were low than expected, DDM that is a relatively flexible aromatic diamine monomer containing flexible methylene linkage was used as a diamine comonomer. Consequently, the copolyimides based on DBAE-8-13 (50\%)/DDM (50\%) were soluble, while the ones based on DBAE-8-13 (25\% or $75 \%$ )/DDM $(75 \%$ or $25 \%)$ were insoluble. It is speculated that the effect of copolymerization for the enhancement of flexibility reach to the maximum at the middle composition; DBAE-8-13 (50\%)/DDM (50\%). All of BTDA/DBAE-14/DDM copolyimides were insoluble, probably due to longer alkyl chain. This phenomenon can be explained by our previous results, showing that the most effective alkyl chain length for the enhancement of solubility is twelve and those having more than twelve methylene units decrease the solubility. ${ }^{29}$ Diamine compositions in the final copolyimides can be calculated from ${ }^{1} \mathrm{H}$ NMR measure- 


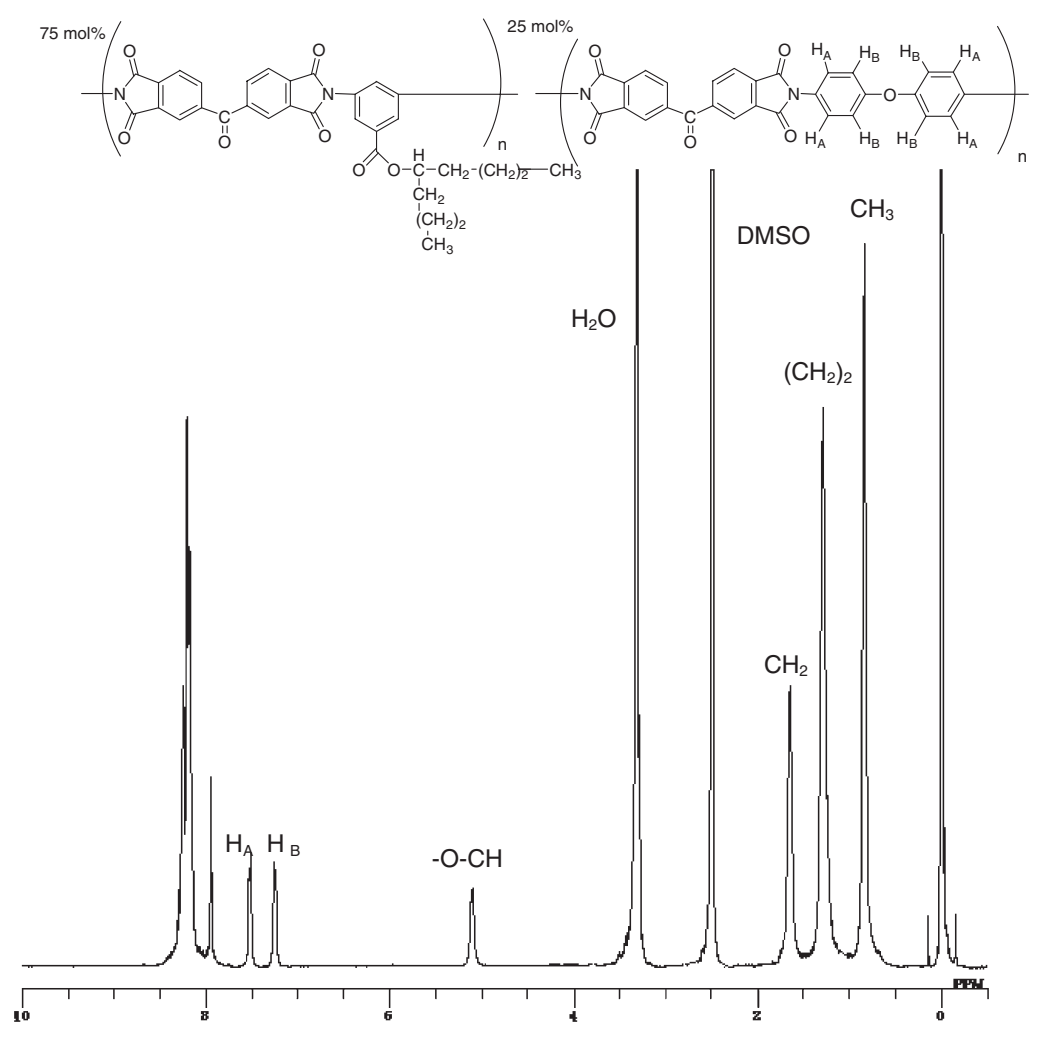

Figure 1. ${ }^{1} \mathrm{H}$ NMR spectrum of a copolyimide based on BTDA/DBAE-9-branch-A/DDE $(100 / 75 / 25) .{ }^{1} \mathrm{H}$ NMR (DMSO- $\left.d_{6}\right): \delta 0.85$ $\left(\mathrm{m}, \mathrm{CH}_{3}\right), 1.30\left(\mathrm{~m},-\left(\mathrm{CH}_{2}\right)_{2} \mathrm{CH}_{3}\right), 1.65\left(\mathrm{~m},-\mathrm{COO}-\left(\mathrm{CHCH}_{2}-\right)_{2}\right), 5.11(\mathrm{~m}, 1 \mathrm{H},-\mathrm{COO}-\mathrm{CH}-), 7.25\left(\mathrm{~d}, \mathrm{H}_{\mathrm{B}}, J=7.4 \mathrm{~Hz}\right), 7.53\left(\mathrm{~d}, \mathrm{H}_{\mathrm{A}}, J=7.4\right.$ $\mathrm{Hz}), 7.95$ (m, ArH), 8.15-8.30 (m, ArH).

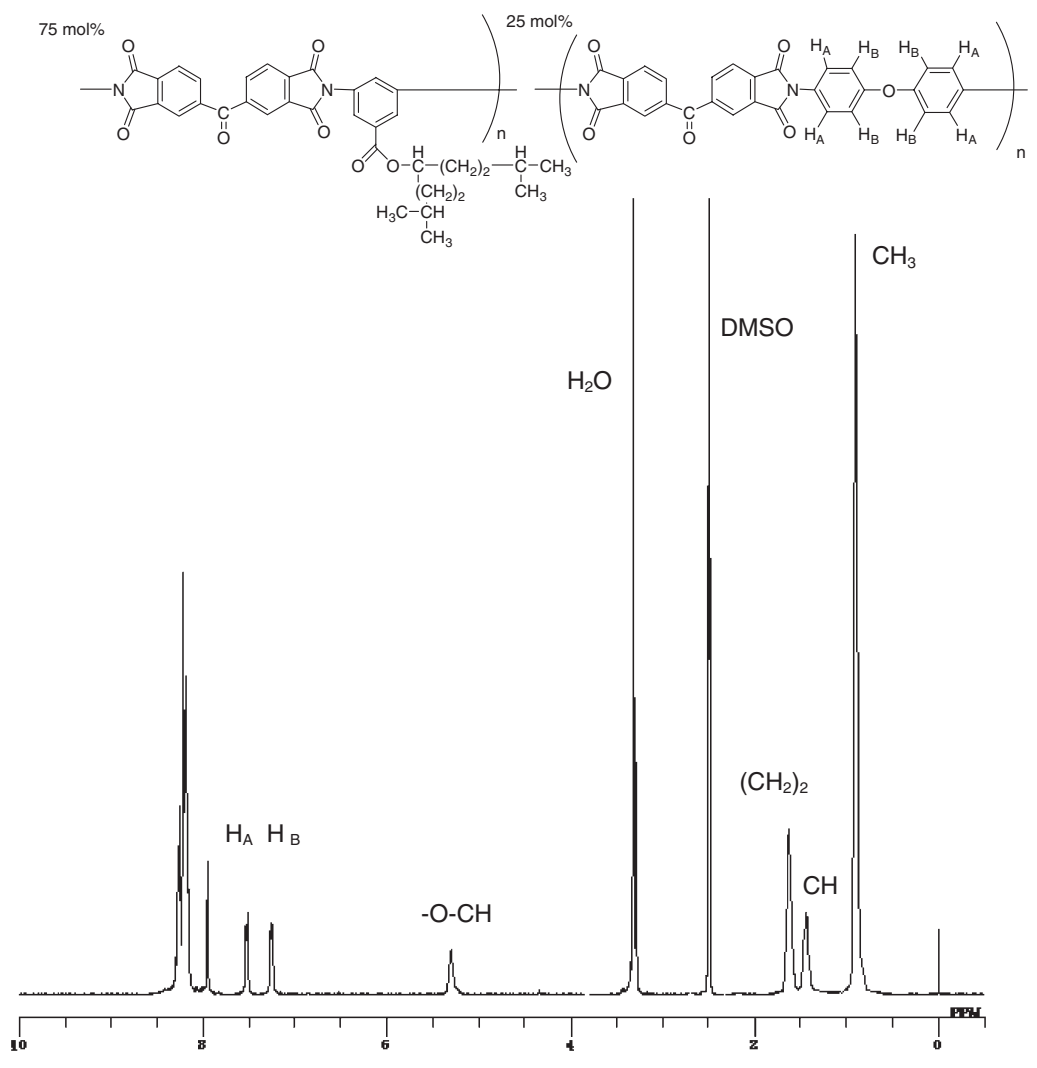

Figure 2. ${ }^{1} \mathrm{H}$ NMR spectrum of a copolyimide based on BTDA/DBAE-9-branch-B/DDE (100/75/25). ${ }^{1} \mathrm{H}$ NMR (DMSO- $\left.d_{6}\right): \delta 0.90$ $\left(\mathrm{m}, \mathrm{CH}_{3}\right), 1.44\left(\mathrm{~m},-\mathrm{CH}-\left(\mathrm{CH}_{3}\right)_{2}\right), 1.62\left(\mathrm{~m},-\mathrm{COO}-\mathrm{CH}-\left(\mathrm{CH}_{2}-\right)_{2}\right), 5.31(\mathrm{~m}, 1 \mathrm{H},-\mathrm{COO}-\mathrm{CH}-), 7.25\left(\mathrm{~d}, \mathrm{H}_{\mathrm{B}}, J=7.4 \mathrm{~Hz}\right), 7.53\left(\mathrm{~d}, \mathrm{H}_{\mathrm{A}}, J=\right.$ $8.0 \mathrm{~Hz}), 7.95$ (m, ArH), 8.15-8.30 (m, ArH). 
Table II. Polyimides and copolyimides based on BTDA/DBAE-X/DDM

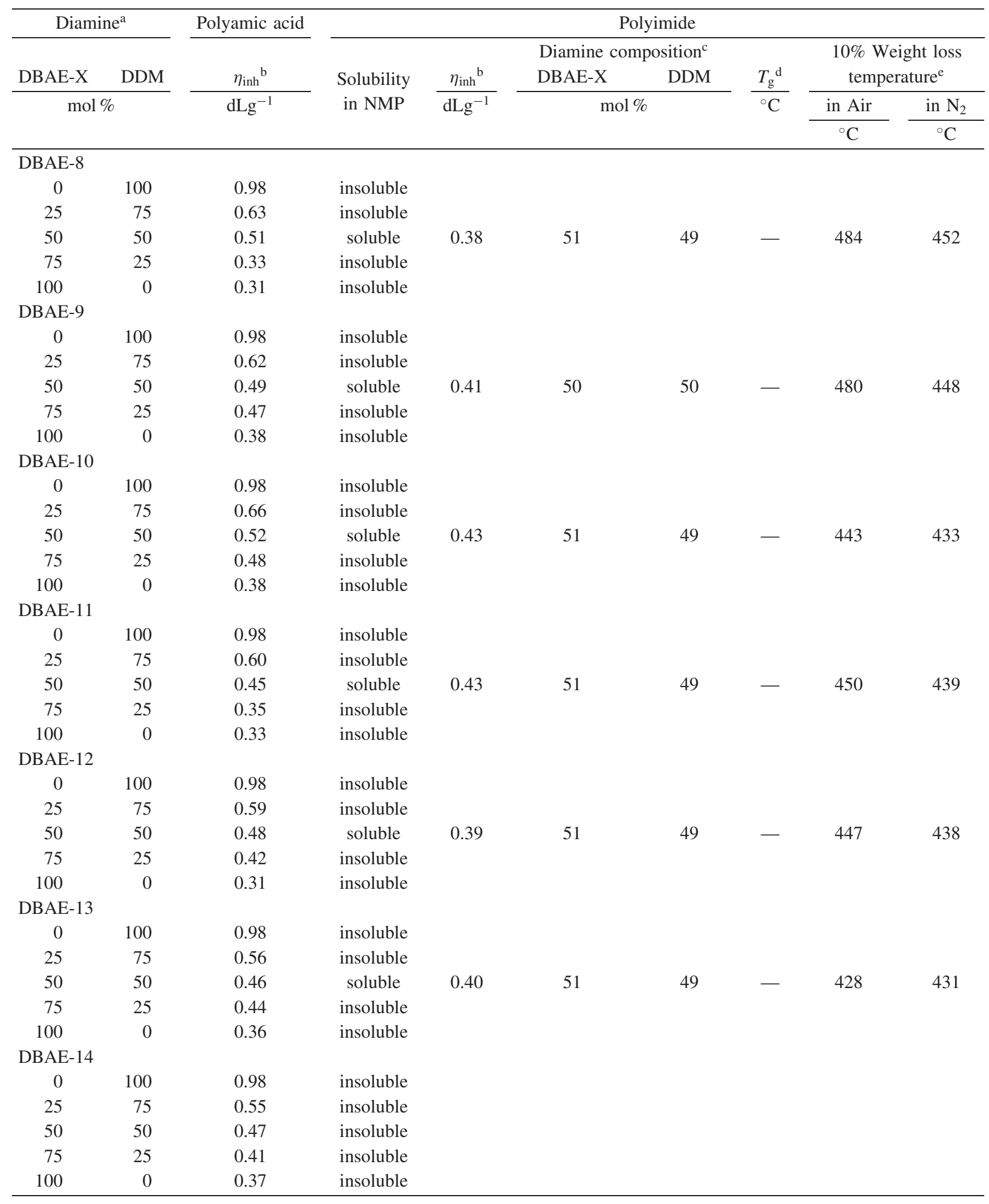

${ }^{\mathrm{a}}$ Equimolar amount of BTDA was used to the total amount of diamine. ${ }^{\mathrm{b}}$ Measured at $0.5 \mathrm{~g} \mathrm{dL}^{-1}$ in NMP at $30^{\circ} \mathrm{C}$. ${ }^{\mathrm{c}}$ Calculated from the intensity ratio of $-\mathrm{OCH}_{2}$-protons based on DBAE-8-13 and methylene protons based on DDM in ${ }^{1} \mathrm{H}$ NMR measurement. ${ }^{\mathrm{d}}$ Measured by DSC at a heating rate of $10{ }^{\circ} \mathrm{C} / \mathrm{min}$ in $\mathrm{N}_{2}$ on second heating. ${ }^{e}$ Measured by TGA at a heating rate of $10^{\circ} \mathrm{C} / \mathrm{min}$.

ment in pyridine- $d_{5}$ (Table II, Figure 3 ). Specifically, the diamine compositions, DBAE-X/DDM were calculated from the intensity ratio of $-\mathrm{OCH}_{2}$-proton based on DBAE-8-13 and methylene protons based on DDM, and in a fair agreement with the initial monomer compositions (Table II). Inherent viscosities $\left(\eta_{\text {inh }}\right)$ of copolyimides based on DBAE-8-13 $(50 \%) / \mathrm{DDM}(50 \%)$ were relatively lower in the range 


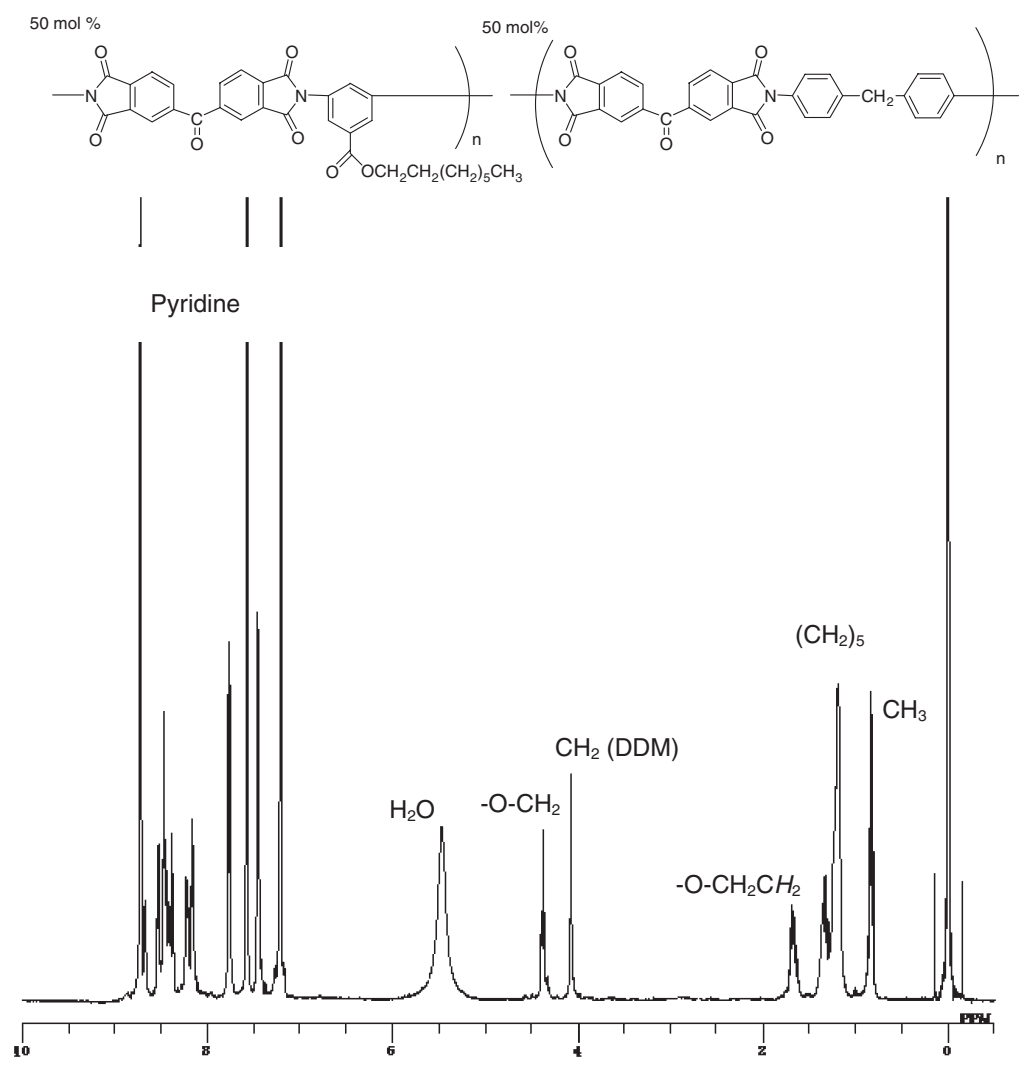

Figure 3. ${ }^{1} \mathrm{H}$ NMR spectrum of a copolyimide based on BTDA/DBAE-8/DDM (100/50/50). ${ }^{1} \mathrm{H}$ NMR (pyridine- $\left.d_{5}\right): \delta 0.83(\mathrm{t}, J=$ $6.80 \mathrm{~Hz}), 1.19\left(\mathrm{~m}, 12 \mathrm{H},-\left(\mathrm{CH}_{2}\right)_{5}-\mathrm{CH}_{3}\right), 1.69\left(\mathrm{~m},-\mathrm{COOCH}_{2} \mathrm{CH}_{2}-\right), 4.08\left(\mathrm{~s}, \mathrm{CH}_{2}\right.$ of DDM), $4.38\left(\mathrm{t},-\mathrm{COOCH}_{2} \mathrm{CH}_{2}-, J=6.80 \mathrm{~Hz}\right), 7.44-$ $8.73\left(\mathrm{~m}\right.$, ArH overlapped with impurities of pyridine- $\left.d_{5}\right)$.

of $0.38-0.43 \mathrm{dLg}^{-1}$ in comparison with copolyimide based on DBAE-9-branch-A(B)/DDE probably due to the relatively lower reactivity of DDM that was previously reported. ${ }^{25}$ However, copolyimides based on DBAE-8-13 (50\%)/DDM (50\%) still show the good film formation ability.

\section{Polymer Properties}

The glass transition temperatures $\left(T_{\mathrm{g}}\right)$ were determined by DSC measurements and the thermal stabilities of these polyimides were evaluated by $10 \%$ weight-loss temperatures $\left(\mathrm{Td}_{10}\right)$ in TGA measurements (Table I, II). The representative TGA traces are shown in Figures 4-6. $T_{\mathrm{g}}$ of polyimides and copolyimides based on DBAE-9-branch $\mathrm{A}(\mathrm{B})$ were in the range of $278-296^{\circ} \mathrm{C}$, while the ones based on DBAE-8-13 (50\%)/DDM (50\%) were not recognized. It is speculated that the flexible moieties based on branched alkyl groups contribute the appearance of $T_{\mathrm{g}} \cdot \mathrm{Td}_{10}$ of polyimides based on polyimides and copolyimides based on DBAE-9-branch $\mathrm{A}(\mathrm{B})$ were relatively lower and in the range of $323-348^{\circ} \mathrm{C}$ in air and $327-351{ }^{\circ} \mathrm{C}$ under nitrogen, while these based on DBAE-8-13 (50\%)/DDM (50\%) were regular values that were observed in soluble polyimides based on lateral alkyl chains $\left(\mathrm{ca} .400-500^{\circ} \mathrm{C}\right)$ and in the range of $428-484{ }^{\circ} \mathrm{C}$ in air and $431-452^{\circ} \mathrm{C}$ under nitrogen.
From the fact that steep weight loss was observed in TGA traces of polyimides and copolyimides based on DBAE-9-branch $\mathrm{A}(\mathrm{B})$ at $c a .300^{\circ} \mathrm{C}$ (Figures 4 and $5)$, it is speculated that the degradation of branched alkyl groups containing weak tertiary $\mathrm{C}-\mathrm{H}$ bonds took place. However, it is conceivable that these polyimides having branched alkyl side chains still belong to heat resistant polymers because there are no weight loss up to $300^{\circ} \mathrm{C}$ both in air and under nitrogen.

The solubility of the obtained polyimides was determined in 9 common solvents at $5 \mathrm{wt} \%$ concentration (Table III). These polyimides were soluble in various polar solvents in addition to NMP, however, the solubility depended on the structure of polyimides. In general, the polyimides based on DBAE-8-13 (50\%)/ DDM (50\%) were more soluble in NMP, DMF, DMI and $m$-cresol than the ones based on DBAE-9branch $\mathrm{A}(\mathrm{B})$. On the other hand, the polyimides based on DBAE-8-13 (50\%)/DDM (50\%) were less soluble in DMAc and DMSO. Among branched DBAE type A and $B$, type $B$ seem to be more effective for the enhancement of solubility from the fact that polyimides based on DBAE-9-branch-B were soluble in various polar solvents such as NMP, DMF, DMAc, DMSO, DMI, $m$-cresol, even in non polar solvent, $\mathrm{CH}_{2} \mathrm{Cl}_{2}$, while polyimides based on DBAE-9-branch-A were soluble only in NMP, DMI, $m$-cresol except BTDA/ 


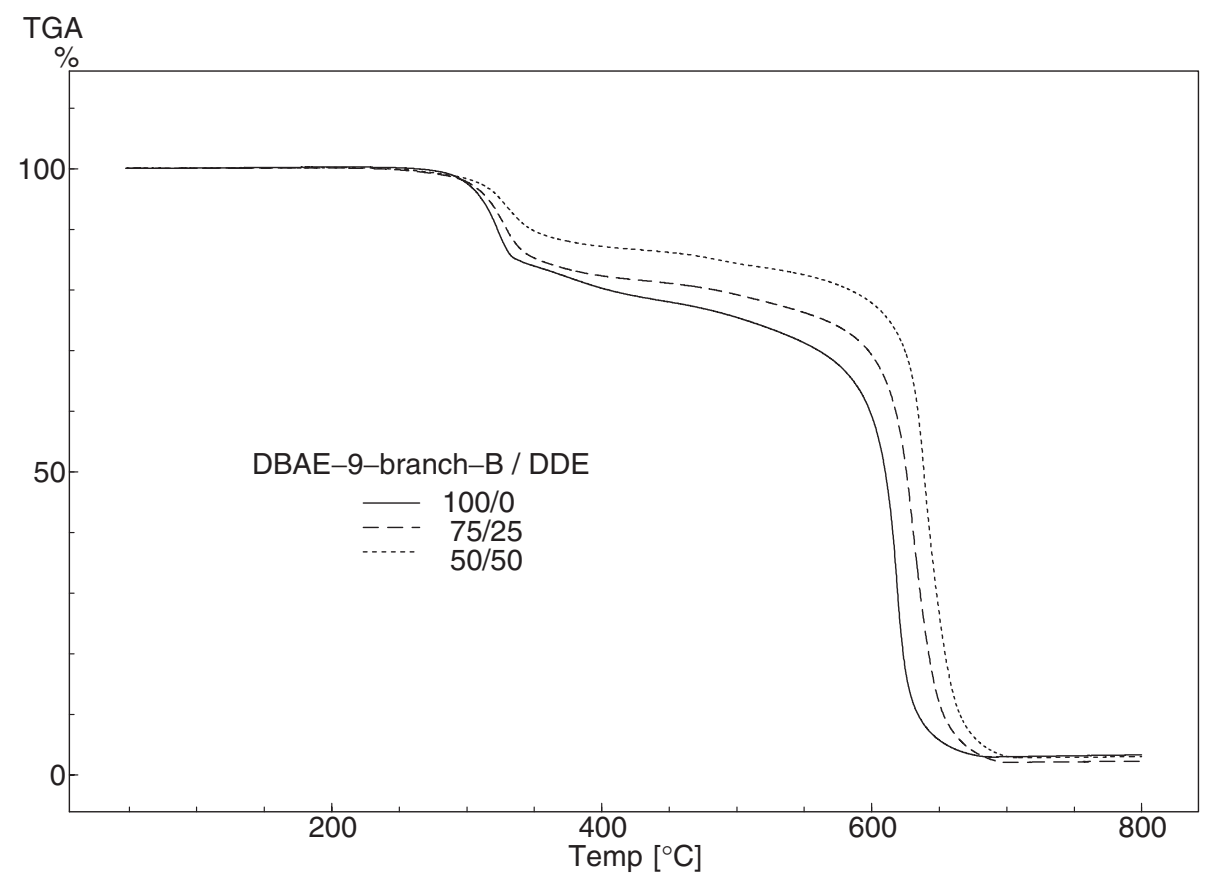

Figure 4. TGA curves of soluble polyimides and copolyimides based on DBAE-9-branch-B in air (BTDA/DBAE-9-branch-B/DDE).

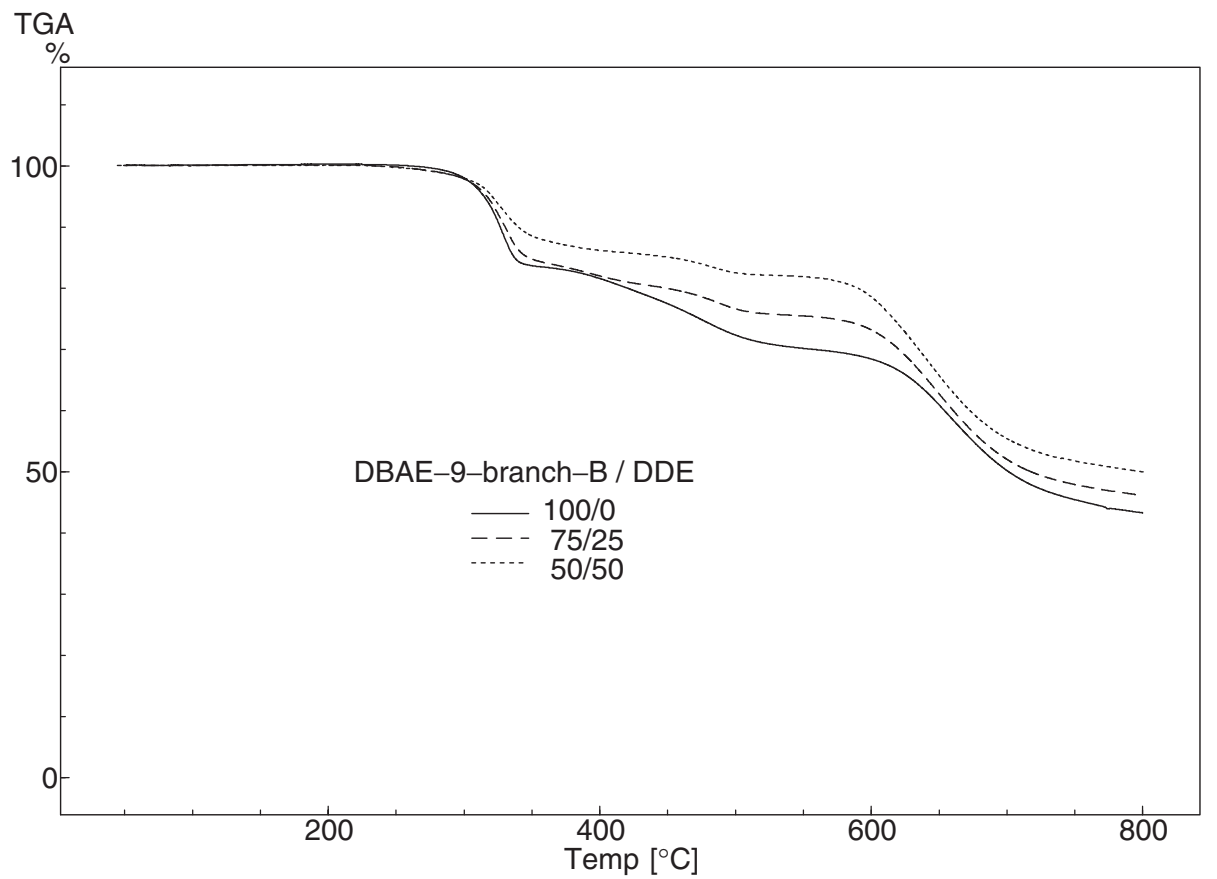

Figure 5. TGA curves of soluble polyimides and copolyimides based on DBAE-9-branch-B under nitrogen (BTDA/DBAE-9branch-B/DDE).

DBAE-9-branch-A/DDE (100/75/25). It can be imagined that these differences of solubility are due to the degree of branch, that is type-B is more branched than type-A.

\section{CONCLUSION}

The synthesis and characterization of a novel series of soluble polyimides, which are based on diamino- benzoic acid alkylester (DBAE) having long-chain alkyl groups with 8-14 carbon atoms, are described. Polyimides obtained from 3,3',4,4'-benzophenonetetracarboxylic dianhydride (BTDA) and DBAE-814, and copolyimides based on BTDA, DBAE-8-14, and 4,4'-diaminodiphenylether (DDE) were insoluble in polar solvents such as $N$-methyl-2-pyrrolidone (NMP) and the effect of long-chain linear alkyl groups for the enhancement of solubility was not recognized. 


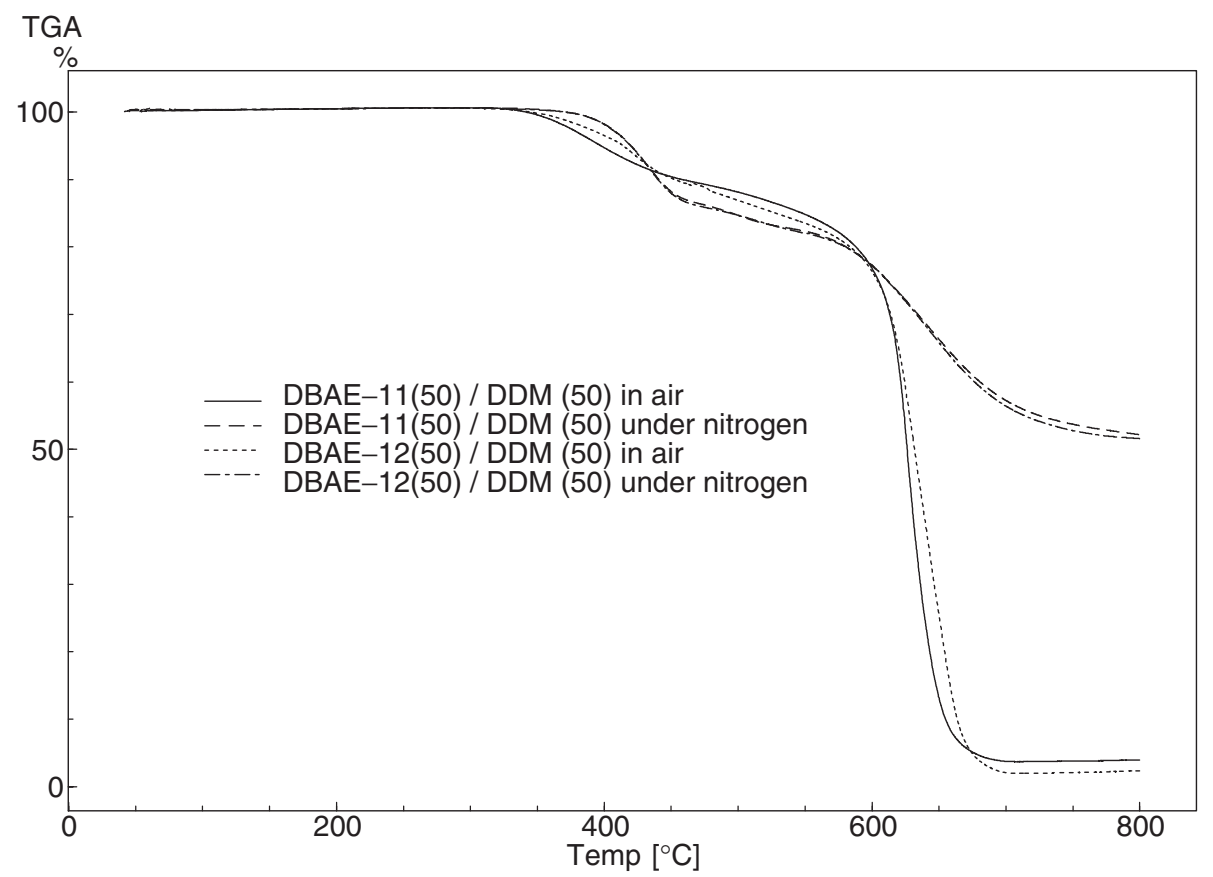

Figure 6. TGA curves of representative soluble copolyimides based on BTDA/DBAE-X/DDM (100/50/50).

Table III. Solubility behavior of polyimides and copolyimides based on BTDA/DBAE-X/DDE and BTDA/DBAE-X/DDM in various solvents

\begin{tabular}{|c|c|c|c|c|c|c|c|c|c|c|}
\hline \multirow{2}{*}{\multicolumn{2}{|c|}{$\begin{array}{c}\text { Diamines } \\
\mathrm{mol} \%\end{array}$}} & \multicolumn{9}{|c|}{ Solubility $^{\mathrm{a}}$} \\
\hline & & \multirow[t]{2}{*}{ NMP } & \multirow[t]{2}{*}{$\mathrm{DMF}$} & \multirow[t]{2}{*}{ DMAc } & \multirow[t]{2}{*}{ DMSO } & \multirow[t]{2}{*}{ DMI } & \multirow[t]{2}{*}{$m$-Cresol } & \multirow[t]{2}{*}{ THF } & \multirow[t]{2}{*}{$\mathrm{CH}_{2} \mathrm{Cl}_{2}$} & \multirow[t]{2}{*}{$\mathrm{H}_{2} \mathrm{SO}_{4}$} \\
\hline DBAE-9-branch-A & DDE & & & & & & & & & \\
\hline 50 & 50 & $\mathrm{~S}(\mathrm{~h})$ & I & I & $\mathrm{I}$ & $\mathrm{PS}(\mathrm{h})$ & PS & I & I & $\mathrm{S}$ \\
\hline 75 & 25 & $\mathrm{~S}(\mathrm{~h})$ & $\mathrm{PS}(\mathrm{h})$ & $\mathrm{PS}(\mathrm{h})$ & $\mathrm{S}(\mathrm{h})$ & $\mathrm{S}(\mathrm{h})$ & $\mathrm{S}$ & I & I & S \\
\hline 100 & 0 & $\mathrm{~S}(\mathrm{~h})$ & I & I & I & $\mathrm{S}(\mathrm{h})$ & $\mathrm{S}(\mathrm{h})$ & I & I & S \\
\hline DBAE-9-branch-B & DDE & & & & & & & & & \\
\hline 50 & 50 & $\mathrm{~S}(\mathrm{~h})$ & I & $\mathrm{PS}(\mathrm{h})$ & $\mathrm{PS}(\mathrm{h})$ & $S(h)$ & $\mathrm{S}(\mathrm{h})$ & I & I & S \\
\hline 75 & 25 & $\mathrm{~S}$ & $\mathrm{~S}(\mathrm{~h})$ & $\mathrm{S}$ & $\mathrm{S}(\mathrm{h})$ & $\mathrm{S}$ & $\mathrm{S}$ & I & PS & S \\
\hline 100 & 0 & $\mathrm{~S}(\mathrm{~h})$ & $\mathrm{S}(\mathrm{h})$ & $\mathrm{S}(\mathrm{h})$ & $\mathrm{S}(\mathrm{h})$ & $S$ & $S$ & I & I & $S$ \\
\hline DBAE-8 (50) & DDM (50) & $\mathrm{S}(\mathrm{h})$ & PS(h) & I & I & $S$ & $S$ & I & I & $S$ \\
\hline DBAE-9 (50) & DDM (50) & $\mathrm{S}$ & $\mathrm{S}$ & I & I & S & $S$ & I & I & S \\
\hline DBAE-10 (50) & DDM (50) & $\mathrm{S}(\mathrm{h})$ & PS(h) & I & I & S & S & I & I & S \\
\hline DBAE-11 (50) & DDM (50) & $\mathrm{S}(\mathrm{h})$ & $\mathrm{S}(\mathrm{h})$ & I & I & S & S & I & I & S \\
\hline DBAE-12 (50) & DDM (50) & $\mathrm{S}$ & $\mathrm{PS}(\mathrm{h})$ & I & I & $\mathrm{S}$ & S & I & I & S \\
\hline DBAE-13 (50) & DDM (50) & $\mathrm{S}(\mathrm{h})$ & $\mathrm{PS}(\mathrm{h})$ & I & I & $\mathrm{S}(\mathrm{h})$ & S & I & I & S \\
\hline
\end{tabular}

${ }^{a}$ S, soluble; $\mathrm{S}(\mathrm{h})$, soluble after heating; PS, partly soluble; PS(h), partly soluble after heating; I, insoluble.

However, it was found that two methods improve the solubility. One is the use of branched alkyl groups such as nonan-5-yl and 2,6-dimethylheptane-4-yl, and the other is the use of relatively flexible diamine co-monomer, 4,4'-diaminodiphenylmethane (DDM). These polyimides and copolyimides based on DBAE were soluble in various polar solvents and exhibited good thermal stability in air and under nitrogen. The effect of DBAE for the enhancement of solubility was lower than the effects of alkyloxydiaminobenzene (AODB) bearing alkyl groups via an ether linkage and alkyldiaminobenzophenone (ADBP) bearing alkyl groups via a benzoyl linkage, probably due to the rigid ester linkage group in DBAE. According to the fact that polyimides having branched alkyl chains are effective on the enhancement of solubility, polyimides having dendron side chain have been also investigated in our laboratory. ${ }^{30}$ These results contribute the elucidation of structure-properties relation in the studies of soluble polyimides, and give the useful information especially in the field of polyimide alignment films for LCDs that sometime contains alkyl chains for the generation of pretilt angle. 
Acknowledgment. The authors thank Drs. Shuntaro Mataka and Tsutomu Ishi-I of Kyushu University, Japan for various advises. The authors thank many students of Kurume National College of Technology for the help of experiments. Financial support from Cheil Industries Inc. is gratefully acknowledged.

\section{REFERENCES}

1. a) "Polyimides," K. L. Mittal, Ed., Plenum Press, New York N. Y., 1984.

b) "Polyimides," M. K. Ghosh and K. L. Mittal, Ed., Marcel Dekker, New York N. Y., 1996.

2. S.-H. Hsiao and T.-L. Hung, Polym. J., 34, 225 (2002).

3. D.-J. Liaw, H.-P. Hung, D.-N. Hsu, and W.-H. Chen, Polym. J., 34, 307 (2002).

4. J. G. Liu, M. H. He, H. W. Zhou, Z. G. Qian, F. S. Wang, and S. Y. Yang, J. Polym. Sci., Part A: Polym. Chem., 40, 110 (2002).

5. D. S. Reddy, C.-F. Shu, and F.-I. Wu, J. Polym. Sci., Part A: Polym. Chem., 40, 262 (2002).

6. C.-P. Yang and R.-S. Chen, J. Polym. Sci., Part A: Polym. Chem., 40, 429 (2002).

7. C.-P. Yang, S.-H. Hsiao, and M.-F. Hsu, J. Polym. Sci., Part A: Polym. Chem., 40, 524 (2002).

8. Y. S. Kim and J. C. Jung, J. Polym. Sci., Part A: Polym. Chem., 40, 1764 (2002).

9. D.-J. Liaw, C.-Y. Hsu, P.-N. Hsu, and S.-L. Lin, J. Polym. Sci., Part A: Polym. Chem., 40, 2066 (2002).

10. Y. Watanabe, Y. Sasaki, Y. Shibasaki, S. Ando, M. Ueda, Y. Oishi, and K. Mori, Macromolecules, 35, 2277 (2002).

11. D. S. Thompson, D. W. Thompson, and R. E. Southward, Chem. Mater., 14, 30 (2002).

12. K. Kudo, D. Nonokawa, J. Li, and S. Shiraishi, J. Polym. Sci., Part A: Polym. Chem., 40, 4038 (2002).

13. B. Y. Myung, J. J. Kim, and T. H. Yoon, J. Polym. Sci., Part A: Polym. Chem., 40, 4217 (2002).

14. Y.-H. Kim, S.-K. Ahn, H.-S. Kim, and S.-K. Kwon, J. Polym. Sci., Part A: Polym. Chem., 40, 4288 (2002).

15. T.-S. Leu and C.-S. Wand, J. Appl. Polym. Sci., 87, 945 (2003).

16. H. Fan, Y. Gu, and M. Xie, J. Polym. Sci., Part A: Polym. Chem., 41, 554 (2003).

17. H.-S. Kim, Y.-H. Kim, S.-K. Ahn, and S.-K. Kwon, Macromolecules, 36, 2327 (2003).
18. C.-P. Yang, R.-S. Chen, and K.-H. Chen, J. Polym. Sci., Part A: Polym. Chem., 41, 922 (2003).

19. C.-P. Yang, R.-S. Chen, and H.-C. Chiang, Polym. J., 35, 662 (2003).

20. S.-H. Hsiao, C.-L. Chung, and M.-L. Lee, J. Polym. Sci., Part A: Polym. Chem., 42, 1008 (2004).

21. C. Gao, X. Wu, G. Lv, M. Ding, and L. Gao, Macromolecules, 37, 2754 (2004).

22. D.-J. Liaw and F.-C. Chang, J. Polym. Sci., Part A: Polym. Chem., 42, 5766 (2004).

23. S.-H. Hsiao and K.-H. Lin, J. Polym. Sci., Part A: Polym. Chem., 43, 331 (2005).

24. B. Liu, W. Hu, T. Matsumoto, Z. Jiang, and S. Ando, J. Polym. Sci., Part A: Polym. Chem., 43, 3018 (2005).

25. Y. Tsuda, Y. Tanaka, K. Kamata, N. Hiyoshi, S. Mataka, Y. Matsuki, M. Nishikawa, S. Kawamura, and N. Bessho, Polym. J., 29, 574 (1997).

26. Y. Tsuda, K. Etou, N. Hiyoshi, M. Nishikawa, Y. Matsuki, and N. Bessho, Polym. J., 30, 222 (1998).

27. Y. Tsuda, R. Kuwahara, K. Fukuda, K. Ueno, and J.-M. Oh, Polym. J., 37, 126 (2005).

28. Y. Tsuda, T. Kawauchi, N. Hiyoshi, and S. Mataka, Polym. J., 32, 594 (2000).

29. Y. Tsuda, K. Kanegae, and S. Yasukouchi, Polym. J., 32, 941 (2000).

30. Y. Tsuda, R. Kuwahara, and J.-M. Oh, Trans. Mater. Res. Soc. Jpn., 29, 267 (2004).

31. Y. Tsuda, T. Yoshida, and T. Kakoi, Polym. J., 38, 88 (2005).

32. J. R. Sasthav and F. W. Harris, Polymer, 36, 4911 (1995).

33. K.-W. Lee, S.-H. Paek, A. Lien, C. Durning, and H. Fukuro, Macromolecules, 29, 8894 (1996).

34. S.-H. Paek, C. J. Durning, K.-W. Lee, and A. Lien, J. Appl. Phys., 83, 1270 (1988).

35. H. Wang, Z. Shen, M. Guo, S. Z. D. Cheng, and F. W. Harris, Polym. Prepr. (Am. Chem. Soc., Div. Polym. Chem.), 40(2), 884 (1999).

36. Y. Tsuda, Polym. Prepr. (Am. Chem. Soc., Div. Polym. Chem.), 40(2), 1215 (1999).

37. L. Li, J. Yin, Y. Sui, H.-J. Xu, J.-H. Fang, Z.-K. Zhu, and Z.-G. Wang, J. Polym. Sci., Part A: Polym. Chem., 38, 1943 (2000).

38. S. W. Lee and M. Ree, J. Polym. Sci., Part A: Polym. Chem., 42, 1322 (2004).

39. S. J. Lee, J. C. Jung, S. W. Lee, and M. Ree, J. Polym. Sci., Part A: Polym. Chem., 42, 3130 (2004). 